\title{
Factores que influencian los cambios en el puntaje de la Escala de Epworth en adultos remitidos a polisomnografía basal en una clínica de sueño del nororiente colombiano
}

\section{Leslie Vargas-Ramirez, Fabio Bolivar, Diana Cano, Katherine Rincón, Maria Camila Gómez, lara Lizarazo, Nicolás Contreras. Instituto Neumológico del Oriente S.A, Bucaramanga, Colombia}

Introducción: La somnolencia diurna es un síntoma importante del síndrome de apnea hipopnea obstructiva del sueño (SAHS). Utilizamos la escala de Epworth como medida objetiva y puede verse influenciada por edad, sexo y comorbilidades del paciente.

Objetivo: evaluar los factores clínicos que se asociaron a cambios en la escala de Epworth en pacientes remitidos para polisomnografía basal en nuestra institución.

Materiales y Métodos: Estudio de corte trasversal retrospectivo en 797 pacientes remitidos a una clínica de sueño durante el 2018. Se realizó un modelo de poisson para evaluar factores clínicos asociados a los cambios esperados en el puntaje de la escala de Epworth para medición de la somnolencia diurna.

Resultados: 797 pacientes con diagnóstico de SAHS por polisomnografía. Los factores antropométricos asociados con un incremento en el puntaje esperado para la escala de somnolencia diurna de Epworth fueron edad, IMC y circunferencia de cuello. En el polisomnograma se encontró asociación con un valor de IAH de 5 $15 / \mathrm{h}$ y con el índice de desaturación. La presencia de diabetes mellitus y depresión se asociaron con cambios en la escala, así como el consumo de bebidas cafeinadas.

Factores asociados a cambios en el puntaje de la Escala de Epworth

\begin{tabular}{|l|c|c|c|}
\hline & IRR & IC 95\% & P \\
\hline Sexo & 0.977 & $0.929-1.026$ & 0.359 \\
\hline Edad & 0.996 & $0.994-0.998$ & $<0.001$ \\
\hline IMC & 1.001 & $1.000-1.002$ & 0.007 \\
\hline Circunferencia Cuello & 1.005 & $1.002-1.009$ & 0.002 \\
\hline IAH 5-15/h & 1.108 & $1.029-1.192$ & 0.006 \\
\hline IAH >15/h & 1.057 & $0.980-1.141$ & 0.148 \\
\hline Consumo de alcohol & 1.038 & $0.990-1.088$ & 0.120 \\
\hline Consumo de cafeína & 1.070 & $1.026-1.116$ & 0.001 \\
\hline ACV & 0.965 & $0.851-1.096$ & 0.591 \\
\hline HTA & 0.981 & $0.934-1.031$ & 0.464 \\
\hline Diabetes mellitus & 1.105 & $1.032-1.183$ & 0.004 \\
\hline Depresión & 0.932 & $0.876-0.991$ & 0.027 \\
\hline ERGE & 1.046 & $0.997-1.097$ & 0.061 \\
\hline Índice de desaturación & 1.001 & $1.000-1.002$ & 0.012 \\
\hline p: nivel de significancia $<0.05$ & & &
\end{tabular}

CONCLUSIONES: Cambios en el puntaje de la escala de Epworth se ven influenciados por factores antropométricos, alteraciones polisomnográficas y comorbilidades. 